however, the role of heterozygous dosage anomalies in PD remains unclear.

Original article Kay DM et al. (2007) Heterozygous parkin point mutations are as common in control subjects as in Parkinson's patients. Ann Neurol 61: 47-54

\section{Antiepileptic drug shows promise for treatment of Parkinson's disease}

Zonisamide is an antiepileptic drug with a long half-life, which has been widely prescribed in Japan. Following several preliminary trials indicating that zonisamide might improve the main symptoms of Parkinson's disease (PD), the Japan Zonisamide on PD Study Group carried out research to examine the efficacy, safety and tolerability of daily oral zonisamide in a cohort of patients with PD who were not responding well to levodopa.

In a multicenter, double-blind, placebocontrolled trial, the researchers randomized 347 patients between 12 weeks of zonisamide at 25,50 or $100 \mathrm{mg} /$ day or placebo. Baseline assessment was conducted after a 2-week run-in period to reduce placebo effects. All patients continued their levodopa treatment.

When compared with those receiving placebo, patients receiving $25 \mathrm{mg}$ or $50 \mathrm{mg}$ doses of zonisamide showed a significant improvement in their Unified Parkinson's Disease Rating Scale Part III total over baseline $(P=0.001$ and $P=0.003$, respectively). In addition, compared with those on placebo, the mean duration of 'off' time was significantly reduced in patients taking $50 \mathrm{mg}$ or $100 \mathrm{mg}$ doses of zonisamide $(P=0.014$ and $P=0.013$, respectively). The changes from baseline with regard to the Modified Hoehn and Yahr Scale were not significantly different between the zonisamide and placebo groups. Although the incidence of adverse effects documented in the $100 \mathrm{mg}$ zonisamide group was markedly higher than that in the other groups, the incidences of hallucination and dyskinesia-of particular concern with antiPD drugs-were similar across all treatment groups. The authors conclude that zonisamide is a safe and well-tolerated adjunctive treatment for patients with PD.

Original article Murata M et al. and The Japan Zonisamide on PD Study Group (2007) Zonisamide improves motor function in Parkinson disease: a randomized, double-blind study. Neurology 68: 45-50

\section{Selective affinity resins remove TSE infectivity from blood}

Transmissible spongiform encephalopathies (TSEs) can be contracted via blood transfusion. Diagnostic testing of blood donations might not be realistic in populations with high exposure to TSEs (e.g. the UK), and consequently alternative protective strategies have been considered. Gregori et al. have investigated the removal of TSE infectious agents from blood, and have concluded that TSE affinity ligands might offer an effective way of limiting or preventing iatrogenic TSE transmission.

A series of increasingly stringent screens identified the ligand L13, which binds both normal and abnormal prion protein. L13 was immobilized on resin in two chromatography columns, which were challenged in series with leukoreduced scrapie-infected hamster whole blood. TSE infection was found in 15 of 99 hamsters 540 days after inoculation with the leukoreduced blood. Flow-through from the first chromatography column infected 2 of 99 hamsters and flow-through from the second column infected 0 of 96 hamsters. The consecutive challenge process reduced endogenous TSE infectivity to below the limit of detection of the bioassay employed (0.2 infectious doses $/ \mathrm{ml})$.

The authors also tested the ligand L13Aa functional equivalent of $L 13$ produced at a manufacturing scale. L13A removed TSE infectivity from leukoreduced hamster whole blood with comparable efficacy to L13 (i.e. to below the bioassay detection limit after the second flow-through); both ligands have also been shown to adsorb prion protein from human blood. A filter device (P-CAPT ${ }^{\circledR}$, MacoPharma, Mouvaux, France) incorporating L13A has been produced for reduction of TSE infectivity in leukoreduced red blood cell concentrates.

\footnotetext{
Original article Gregori L et al. (2006) Reduction in infectivity of endogenous transmissible spongiform encephalopathies present in blood by adsorption to selective affinity resins. Lancet 368: 2226-2230
}

\section{Report on a case of vCJD with probable iatrogenic origins}

Since 1997 in the UK it has been possible to identify patients who have received blood components from donors who subsequently developed variant Creutzfeldt-Jakob disease 